\title{
High-Resolution Algorithm for Image Segmentation in the Presence of Correlated Noise
}

\author{
Haiping Jiang, Salah Bourennane, and Caroline Fossati \\ Institut Fresnel/UMR CNRS 6133, Ecole Centrale Marseille, D.U. de Saint-Jérôme, 13397 Marseille Cédex 20, France
}

Correspondence should be addressed to Salah Bourennane, salah.bourennane@fresnel.fr

Received 23 July 2009; Accepted 14 December 2009

Academic Editor: Jiri Jan

Copyright () 2010 Haiping Jiang et al. This is an open access article distributed under the Creative Commons Attribution License, which permits unrestricted use, distribution, and reproduction in any medium, provided the original work is properly cited.

Multiple line characterization is a most common issue in image processing. A specific formalism turns the contour detection issue of image processing into a source localization issue of array processing. However, the existing methods do not address correlated noise. As a result, the detection performance is degraded. In this paper, we propose to improve the subspace-based high-resolution methods by computing the fourth-order slice cumulant matrix of the received signals instead of second-order statistics, and we estimate contour parameters out of images impaired with correlated Gaussian noise. Simulation results are presented and show that the proposed methods improve line characterization performance compared to second-order statistics.

\section{Introduction}

It has been shown that a specific signal generation process yields, when applied to an image containing multiple lines, to signals which follow an array processing model. Then high-resolution methods, such as MUSIC [1] and ESPRIT [2] algorithms, are used. Subspace-based Line DEtection (SLIDE) algorithm [3] and recently other methods [4] have been proposed, which can be effectively exploited to estimate line parameters (orientation and offset).

However, these methods assume that the noise is white and are based exclusively on second-order statistics. When the noise is correlated, these developed algorithms are not efficient.

That is the fact that correlated noise exists in several circumstances. For instance, correlated noise occurs in many imaging systems such as scanners and push-broom imagers. In medical X-ray imaging, while noise in an X-ray beam is uncorrelated, it is correlated in observed images. This is due to the detectors, where X-ray photons typically evoke multiple light photons that can contribute to different pixels.

In Section 2, we first model the image data and formulate the problem by introducing cumulant-based model. In Section 3, high-resolution algorithms for estimating line orientation, which are MUSIC-like algorithm and improved TLS-ESPRIT algorithm, are derived from fourth-order cumulant of the signal realizations received by virtual array sensors. After orientation estimation, the extension of Hough transform is applied to find a line offset. In Section 4, numerical simulations are presented to show that cumulantbased methods are efficient when the processed image is impaired by correlated noise.

\section{Problem Formulation}

2.1. Image Data Model. Let us consider 2-dimensional binary image $I(x, y)$ (see Figure 1), where a line is characterized by its $x$-axis offset and the angle that it makes with the normal to the $x$-axis at the interception point, with positive angles defined for lines having positive $y$-axis offsets. The "1"-valued pixels represent useful pixels and form the outline of the estimated contour, while the background is demonstrated by "0"-valued pixels. The image consists of $\mathrm{N}$ rows and $\mathrm{C}$ columns in Figure 1. In order to make the image data representation similar to sensor array processing, array sensors are supposed to be placed aside the image, each sensor receiving signals only from its corresponding row in the matrix [3]. All the pixels in image are assumed to propagate narrowband electromagnetic waves with zero initial phases. Furthermore, assume that the waves emanating from pixels in a given row of the image matrix are confined to 


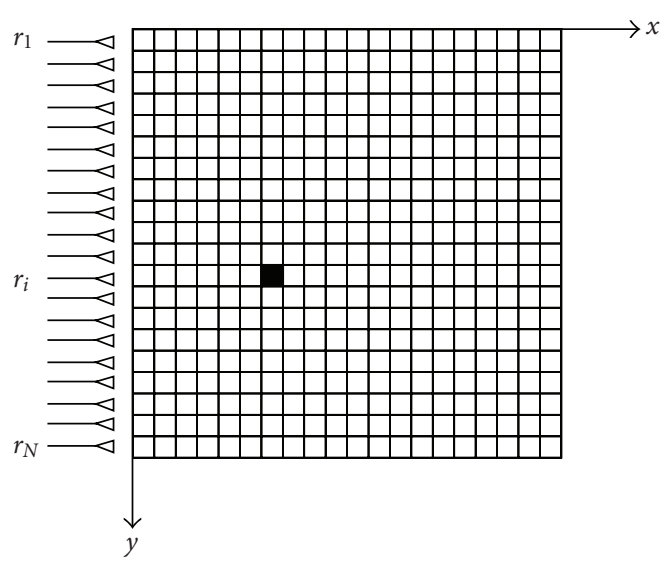

(a)

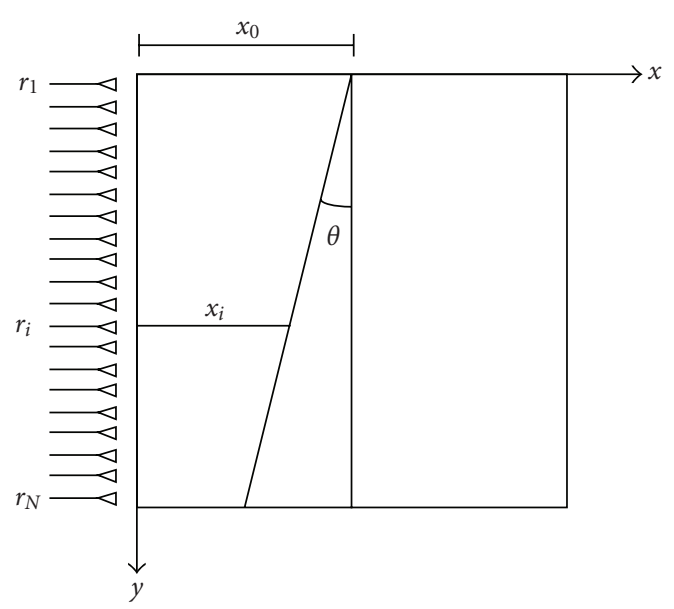

(b)

FIGURE 1: image data model.

travel only along that row towards the corresponding sensor. In this propagation environment, each straight line in the image will in effect be equivalent to a wavefront of a traveling plane wave. For this purpose some signals are generated out of the image data. When there is only one line in image and no outlier pixels, the signal received by each sensor is: $r_{i}=e^{-j \mu x_{i}}(i=1, \ldots, N)$, where $\mu$ is a propagation parameter [3] and $x_{i}$ is the $x$-axis offset in ith row, $x_{i}=x_{0}-(i-1) \tan (\theta)$.

If there are $d$ straight lines and also outlier pixels in image, the signal received by each sensor can be extended

$$
r_{i}=\sum_{k=1}^{d} e^{-j \mu x_{i}}=\sum_{k=1}^{d} e^{-j \mu x_{0}} e^{j \mu(i-1) \tan \left(\theta_{k}\right)}+n(i),
$$

where $n(i)$ is the effect of noise in the $i$ th row. We denote $a\left(\theta_{k}\right)=e^{j \mu(i-1) \tan \left(\theta_{k}\right)}$ and $s_{k}=e^{-j \mu x_{0}}$, at the same time, consider that the image is impaired by the noise, we can be express all the signals in matrix form

$$
\mathbf{r}=\mathbf{A}(\theta) \cdot \mathbf{s}+\mathbf{N}
$$

where $\mathbf{r}=\left[r_{1}, r_{2}, \ldots, r_{N}\right]^{T}, \mathbf{s}=\left[s_{1}, s_{2}, \ldots, s_{d}\right]^{T}$, and $\mathbf{A}(\theta)=\left[\mathbf{a}\left(\theta_{1}\right), \mathbf{a}\left(\theta_{2}\right), \ldots, \mathbf{a}\left(\theta_{d}\right)\right]$ with $k$ th column $\mathbf{a}\left(\theta_{\mathrm{k}}\right)=$ $\left[1, e^{j \mu \tan \left(\theta_{k}\right)}, \ldots, e^{j \mu(N-1) \tan \left(\theta_{k}\right)}\right]^{T}$, therein “ $T$ ” denotes transposition operation.

2.2. Fourth-Order Cumulant-Based Model. For complex variable, the fourth-order cumulant can be generally defined as

$$
\begin{aligned}
\operatorname{cum}_{4}\left(x_{1}, x_{2}, x_{3}^{*}, x_{4}^{*}\right) & \\
= & \mathrm{E}\left\{x_{1} x_{2} x_{3}^{*} x_{4}^{*}\right\}-\mathrm{E}\left\{x_{1} x_{3}^{*}\right\} \mathrm{E}\left\{x_{2} x_{4}^{*}\right\} \\
& -\mathrm{E}\left\{x_{1} x_{4}^{*}\right\} \mathrm{E}\left\{x_{2} x_{3}^{*}\right\}-\mathrm{E}\left\{x_{1} x_{2}\right\} \mathrm{E}\left\{x_{3}^{*} x_{4}^{*}\right\},
\end{aligned}
$$

where “*” denotes conjugate operation. Quite often the complex random variables are analytical signal, so the fourth item of the right side is identically zero. Note that the definition of cumulant of complex random variables is nonunique. In practical terms, continuous signals are often turned into discrete time-series to calculate fourth-order cumulant.

Providing that there is only one source signal $s_{i}(p)$, that is, only one line in image data, the signals received by array sensors is defined as $\mathbf{r}_{i}(p)$. So for all the source signals, the received signals at the $p$ th snapshot are $\mathbf{r}=\sum_{i=1}^{d} \mathbf{r}_{i}(p)=$ $\sum_{i=1}^{d} \mathbf{a}\left(\theta_{i}\right) \cdot s_{i}(p)$, where $s_{i}(p)=e^{j(p-1) \mu \tan \theta_{i}} \cdot e^{-j \mu x_{0_{i}}}(p=$ $1, \ldots, d)$ is the $i$ th source signal at $p$ th snapshot.

We define the vector $\mathbf{y}_{i}(p)$ by

$$
\begin{aligned}
\mathbf{y}_{i}(p) & =\mathbf{r}_{i}(p) \otimes \mathbf{r}_{i}^{H}(p) \\
& =\left(\mathbf{a}\left(\theta_{i}\right) \otimes \mathbf{a}^{*}\left(\theta_{i}\right)\right) \cdot s_{i}(p) s_{i}^{*}(p),
\end{aligned}
$$

where “ $\otimes$ " denotes Kronecker product. Based on the independence property of cumulant and that the fourth-order cumulants of the Gaussian noise are identically zero $[5,6]$, we can get the following fourth-order cumulant of image data after some calculations:

$$
\begin{aligned}
\mathbf{C}_{\mathbf{r r}, 4}= & \sum_{i=1}^{d}\left(\mathbf{a}\left(\theta_{i}\right) \otimes \mathbf{a}^{*}\left(\theta_{i}\right)\right) \\
& \cdot \operatorname{cum}_{4}\left(s_{i}(p), s_{i}(p), s_{i}^{*}(p), s_{i}^{*}(p)\right) \\
& \cdot\left(\mathbf{a}\left(\theta_{i}\right) \otimes \mathbf{a}^{*}\left(\theta_{i}\right)\right)^{H}
\end{aligned}
$$

To make the analogy of the formulation as sensor array processing, we do not have time-dependent measurement signal vectors, because the signals generated by image data is deterministic. There is only a single snapshot across a very large array (see (2)). Therefore, to exploit high-resolution methods with fourth-order cumulant in array processing to image signals, the signals received by array sensors in image 
model are considered to be spatially smoothed and a new matrix $\mathbf{R}_{P}$ is rearranged as

$$
\begin{aligned}
\mathbf{R}_{P} & =\left[\begin{array}{llll}
\mathbf{r}(1) & \mathbf{r}(2) & \cdots & \mathbf{r}(P)
\end{array}\right]=\left[\begin{array}{c}
\mathbf{z}_{1} \\
\vdots \\
\mathbf{z}_{M}
\end{array}\right] \\
& =\left[\begin{array}{cccc}
r_{1} & r_{2} & \cdots & r_{N-M+1} \\
\vdots & \vdots & \ddots & \vdots \\
r_{M} & r_{M+1} & \cdots & r_{N}
\end{array}\right],
\end{aligned}
$$

where $M=N-P+1$ and $d<M \leq N-d+1$. For every snapshot, $\mathbf{r}(p)=\mathbf{A}(\theta) \mathbf{s}(p)+\mathbf{n}(p)(p=1, \ldots, P)$, where $\mathbf{A}(\theta)$ is $M \times d$ matrix and $\mathbf{n}(p)$ is the noise vector acquired by the same rearrangement of the snapshot vectors. Then, all the signals generated by image data can be written in matrix form

$$
\mathbf{R}_{P}=\mathbf{A}(\theta) \mathbf{S}_{P}+\mathbf{N}_{P},
$$

where $\boldsymbol{S}_{P}=[\mathbf{s}(1), \ldots, \mathbf{s}(P)]$ and $\mathbf{N}_{P}=[\mathbf{n}(1), \ldots, \mathbf{n}(P)]$. In this way, a single snapshot across a very large array in (2) is transformed to $P$ snapshots. Now we are ready to get the similar signal as in sensor array processing.

\section{Detection Algorithms}

\subsection{Angle Estimation}

3.1.1. MUSIC-Like Algorithm. In order to reduce the computational load, a slice cumulant matrix (e.g., $\mathrm{C}_{\mathrm{rr}, 4}^{\prime}$, here the first row of $\mathbf{C}_{\mathbf{r r}, 4}$ ), instead of above-mentioned cumulant (see (5)), and offers the same properties [5]

$$
\begin{aligned}
\mathbf{C}_{\mathbf{r r}, 4}^{\prime} & =\left\{\operatorname{cum}_{4}\left(z_{1}(p), z_{i}(p), z_{1}^{*}(p), z_{j}^{*}(p)\right)\right\}, \\
& =\mathbf{A}(\theta) \cdot \Gamma \cdot \mathbf{A}^{H}(\theta), \\
& i=1, \ldots, M ; j=1, \ldots, M ; p=1, \ldots, P .
\end{aligned}
$$

where $z_{i}(p)$ is the signal received by $i$ th senor at $p$ th snapshot, and $\Gamma=\operatorname{diag}\left(\operatorname{cum}_{4}\left(s_{1}(p), s_{1}^{*}(p), s_{1}(p)\right.\right.$, $\left.\left.s_{1}^{*}(p)\right), \ldots, \operatorname{cum}_{4}\left(s_{d}(p), s_{d}^{*}(p), s_{d}(p), s_{d}^{*}(p)\right)\right)$, the diagonal elements of which are the kurtosis. Equation (8) shows that there is no noise term in the cumulant slice matrix computed out of the generated signals. Therefore, when this matrix is computed, the parameters can be better estimated. Obviously, this reshaped matrix is the Hermitian matrix and its dimension is reduced to $M \times M$ from $M^{2} \times M^{2}$, so the computational load is hugely decreased.

From the definition of matrix $\mathbf{A}(\theta)$ (see (2)), which shows that the columns of $\mathbf{A}(\theta)$ are linearly independent, it is easy to get that $\mathbf{C}_{\mathbf{r r}, 4}^{\prime}$ has the rank of $d$. The MUSIC-like algorithm relies on the singular value decomposition (SVD) of matrix $\mathrm{C}_{\mathrm{rr}, 4}^{\prime}$ :

$$
\mathbf{C}_{\mathbf{r r}, 4}^{\prime}=\left[\mathbf{U}_{1} \mathbf{U}_{2}\right]\left[\begin{array}{cc}
\Lambda & 0 \\
\mathbf{0} & \mathbf{0}
\end{array}\right]\left[\begin{array}{c}
\mathbf{U}_{1}^{H} \\
\mathbf{U}_{2}^{H}
\end{array}\right] .
$$

For the independent sources, the columns of $\mathbf{U}_{1}(M \times d)$ span the signal subspace, and the columns of $\mathbf{U}_{2}(M \times(M-$ d)) span the noise subspace [1], $\boldsymbol{\Lambda}=\operatorname{diag}\left(\lambda_{1}, \lambda_{2}, \ldots, \lambda_{\mathrm{d}}\right)$, where $\lambda_{i}$ is the eigenvalue associating with the $i$ th eigenvector. Hence, $\mathbf{U}_{2}$ is orthogonal to the steering vector of $\mathbf{A}(\theta)$. The pseudo spectrum of the MUSIC method is given by

$$
\operatorname{MUSIC}\left(\theta_{k}\right)=\frac{1}{\left\|\mathbf{a}^{H}\left(\theta_{k}\right) \cdot \mathbf{U}_{2}\right\|^{2}} .
$$

In conclusion, practical application of the algorithm involves the following steps.

(1) Estimate the cumulant matrix by (5), (6), and (8).

(2) Find the eigendecomposition of the cumulant matrix by 8 and get the noise subspace $\mathbf{U}_{2}$. If the number of line is unknown, the Minimum Description Length (MDL) or Akaike Information Criterion (AIC) [7] criterion are used.

(3) Compute $\operatorname{MUSIC}\left(\theta_{k}\right)$ by (10). The straight line orientations are estimated through the research of the maxima of $\operatorname{MUSIC}\left(\theta_{k}\right)$.

3.1.2. Improved TLS-ESPRIT Algorithm. In this subsection, we define a matrix for implementing SVD operation, which is more appropriate to the improved TLS-ESPRIT algorithm and is different from the fourth-order cumulant matrix in MUSIC-like algorithm

$$
\begin{aligned}
\mathbf{C}_{\mathbf{r r}, 4}^{\prime \prime} & =\left\{\operatorname{cum}_{4}\left(z_{i}(p), z_{i}(p), z_{i}^{*}(p), z_{j}^{*}(p)\right)\right\}, \\
i & =1, \ldots, N ; j=1, \ldots, N ; p=1, \ldots, P .
\end{aligned}
$$

After spatial smoothing procedure is applied to image data as in (6), the multiple snapshots obtained have length $M$, and $\mathbf{C}_{\mathrm{rr}, 4}^{\prime \prime}$ is also $M \times M$ dimensional matrix.

In order to follow the key steps of TLS-ESPRIT algorithm, we firstly derive the following four $(M-1) \times(M-1)$ submatrices from (11):

$$
\begin{array}{r}
\mathbf{C}_{11}=\left\{\operatorname{cum}_{4}\left(\mathbf{z}_{\mathbf{i}}, \mathbf{z}_{\mathbf{i}}, \mathbf{z}_{\mathbf{i}}^{*}, \mathbf{z}_{\mathbf{j}}^{*}\right)\right\}, \\
i=1, \ldots, M-1 ; j=1, \ldots, M-1, \\
\mathbf{C}_{12}=\left\{\operatorname{cum}_{4}\left(\mathbf{z}_{\mathbf{i}}, \mathbf{z}_{\mathbf{i}}, \mathbf{z}_{\mathbf{i}}^{*}, \mathbf{z}_{\mathbf{j}}^{*}\right)\right\}, \\
i=1, \ldots, M-1 ; j=2, \ldots, M, \\
\mathbf{C}_{21}=\left\{\operatorname{cum}_{4}\left(\mathbf{z}_{\mathbf{i}}, \mathbf{z}_{\mathbf{i}}, \mathbf{z}_{\mathbf{i}}^{*}, \mathbf{z}_{\mathbf{j}}^{*}\right)\right\}, \\
i=2, \ldots, M ; j=1, \ldots, M-1, \\
\mathbf{C}_{22}=\left\{\operatorname{cum}_{4}\left(\mathbf{z}_{\mathbf{i}}, \mathbf{z}_{\mathbf{i}}, \mathbf{z}_{\mathbf{i}}^{*}, \mathbf{z}_{\mathbf{j}}^{*}\right)\right\}, \\
i=2, \ldots, M ; j=2, \ldots, M .
\end{array}
$$

Then, the following matrix can be used to implement SVD operation in place of the covariance matrix:

$$
\mathrm{C}=\left[\begin{array}{ll}
\mathrm{C}_{11} & \mathrm{C}_{12} \\
\mathbf{C}_{21} & \mathbf{C}_{22}
\end{array}\right] \text {. }
$$


The overall improved TLS-ESPRIT algorithm-based cumulant can be summarized as follows.

(1) Form the matrix $\mathbf{C}_{\mathbf{r r}, 4}^{\prime \prime}$ by (5), (6), and (11).

(2) Generate four submatrices from (12) and reconstruct the matrix $\mathbf{C}$ by (13);

(3) Perform SVD operation with $\mathbf{C}$

$$
\mathbf{C}=\mathbf{E} \boldsymbol{\Lambda} \mathbf{E}^{H},
$$

where $\boldsymbol{\Lambda}=\operatorname{diag}\left(\lambda_{1}, \ldots, \lambda_{2(M-1)}\right)$ with the eigenvalues $\lambda_{1} \geq \lambda_{2} \geq \cdots \geq \lambda_{d} \geq \cdots \lambda_{2(M-1)}$ and $\lambda_{1}, \lambda_{2}, \ldots, \lambda_{d}$ corresponding to the signal subspace. Depending on the known conditions, AIC or MDL criterion is used.

(4) Let $\mathbf{E}_{1}$ be the $2(M-1) \times d$ upper-left matrix of $\mathbf{E}$ and let $\mathbf{E}_{2}$ be $2(M-1) \times d$ matrix formed by deleting the first row of matrix E. We carry out the eigendecomposition

$$
\begin{gathered}
{\left[\begin{array}{c}
\mathbf{E}_{1}^{H} \\
\mathbf{E}_{2}^{H}
\end{array}\right]\left[\begin{array}{ll}
\mathbf{E}_{1} & \mathbf{E}_{2}
\end{array}\right]=\mathbf{F} \Lambda_{F} \mathbf{F}^{H}} \\
\mathbf{F}=\left[\begin{array}{ll}
\mathbf{F}_{11} & \mathbf{F}_{12} \\
\mathbf{F}_{21} & \mathbf{F}_{22}
\end{array}\right] .
\end{gathered}
$$

(5) Extract the submatrices $\mathbf{F}_{12}$ and $\mathbf{F}_{22}$, then find the eigenvalues $\left\{\gamma_{k}\right\}$ of $-\mathbf{F}_{12} \mathbf{F}_{22}^{-1}$.

(6) Estimate every line's orientation by

$\theta_{k}=\tan ^{-1}\left(\frac{1}{\mu} \operatorname{Im}\left(\ln \frac{\gamma_{k}}{\left|\gamma_{k}\right|}\right)\right), \quad k=1, \ldots, d$.

3.2. Offset Detection. After we estimate the line's angles, steering matrix $\mathbf{A}(\theta)$ can be computed by (2), so that the maximum likelihood function or other similar methods can be used to find the offsets of lines. In practice, we estimate the offsets using the "Extension of the Hough Transform" [8].

\section{Simulation Results}

In this section, simulation results are presented and the performance of our proposed algorithms is evaluated.

4.1. Correlated Gaussian Noise Simulation. To evaluate realistically the algorithms, correlated Gaussian noise is simulated by the following steps. First, the 2-dimensional random Gaussian noise matrix is generated, which has the same dimensions as the initial binary image and obeys a Gaussian distribution with mean 0 and variance $\sigma^{2}$. Then, let generated Gaussian noise matrix pass the spatial Gaussian low pass filter, so that we can obtain expected correlated Gaussian noise by choosing the filter's parameter. The impulse response of the filter is $h(x, y)=$ $\left(1 / 2 \pi \sigma_{1} \sigma_{2}\right) e^{-\left(x^{2} / 2 \sigma_{1}^{2}\right)-\left(y^{2} / 2 \sigma_{2}^{2}\right)}$, where $\sigma_{1}$ and $\sigma_{2}$ are vertical and horizontal variances, determining the correlation strength of noise image. Specially, $\sigma_{1}=\sigma_{2}$ means an isotropic Gaussian
TABLE 1: Results with increase of CL.

\begin{tabular}{llccc}
\hline & & $\mathrm{CL}=8$ & 12 & 16 \\
\hline \multirow{2}{*}{ ME(MUSIC-like) } & $32^{\circ}$ & 0.2859 & 0.2831 & 0.2801 \\
& $20^{\circ}$ & 0.5358 & 0.2788 & 0.276 \\
Std(MUSIC-like) & $32^{\circ}$ & 0.2858 & 0.2616 & 0.1807 \\
& $20^{\circ}$ & 0.3066 & 0.2815 & 0.2766 \\
ME(TLS-ESPRIT) & $32^{\circ}$ & 0.7885 & 0.3733 & 0.1782 \\
& $20^{\circ}$ & 1.4014 & 0.8247 & 0.3527 \\
Std(TLS-ESPRIT) & $32^{\circ}$ & 1.1963 & 0.6973 & 0.2905 \\
& $20^{\circ}$ & 2.427 & 1.6592 & 0.9817 \\
\hline
\end{tabular}

low pass filter. In the simulation, the filter function $h(x, y)$ should be discretized to get the filter template, which is center symmetric. Herein, the correlation length (CL) of correlated Gaussian noise is defined as $\mathrm{CL}=\left(\mathrm{CL}_{x}+\mathrm{CL}_{y}\right) / 2$, where $\mathrm{CL}_{x}$ and $\mathrm{CL}_{y}$ are $2 \sigma_{1}$ and $2 \sigma_{2}$.

4.2. Algorithm Simulation. We set the size of detected image as $100 \times 100$. As an example in Figure 2, standard variance of white Gaussian noise is 6 and CL of correlated Gaussian noise is 6. For initial image (see Figure 2(a)), there are two straight lines whose angles are $32^{\circ}$ and $20^{\circ}$ and offsets are 95 and 55, respectively. The estimation results provided by cumulant-based MUSIC-like algorithm (see Figure 2(c)) and by the improved TLS-ESPRIT algorithm (see Figure 2(d)) are more accurate than those provided by second-order-based algorithms (see Figure 2(b)). The orientations of two lines are, respectively, $32.3^{\circ}$ and $20.4^{\circ}$ for MUSIC-like algorithm, and $31.8^{\circ}$ and $19.9^{\circ}$ for improved TLS-ESPRIT algorithm. For the offsets, they are, respectively, 93.8 and 52.9 by MUSIC-like algorithm, and 92.6 and 54.8 by TLS-ESPRIT algorithm. Especially when the images are severely corrupted by correlated Gaussian noise, the cumulant-based algorithms still correctly characterize the lines, while the algorithms using second-order statistics yield a large bias.

For $K$ trials, the mean error ME and standard deviation Std are defined like: $\mathrm{ME}=1 / K \sum_{k=1}^{K}\left|x_{i}-\bar{x}\right|$ and Std $=$ $\sqrt{1 / K \sum_{k=1}^{K}\left(x_{i}-\bar{x}\right)^{2}}$, where $x_{k}$ is each estimated angle and $\bar{x}$ is the mean of all estimated angles. From Table 1, we can see that, as the augmentation of correlated noise's CL, it is insensitive to the mean and standard deviation of estimated angle when using MUSIC-like algorithm, compared to the improved TLS-ESPRIT algorithm. Moreover, we also see in the table that the performance of MUSIC-like algorithm is better than improved TLS-ESPRIT algorithm. Since both MUSIC-like algorithm and improved TLS-ESPRIT algorithm should choose the slice cumulant matrices to implement eigen-decomposition, these different choices of the matrices have some impacts on the performance of the algorithms.

The following experiments aim at studying the highresolution capabilities of the proposed algorithm. Firstly, two lines with $15^{\circ}$ and $45^{\circ}$ are considered in the image. The standard variance of white Gaussian noise is set to be 12 , and CL is 16. After we operate 100 trials, the means of estimated angles for MUSIC-like algorithm are, respectively, 


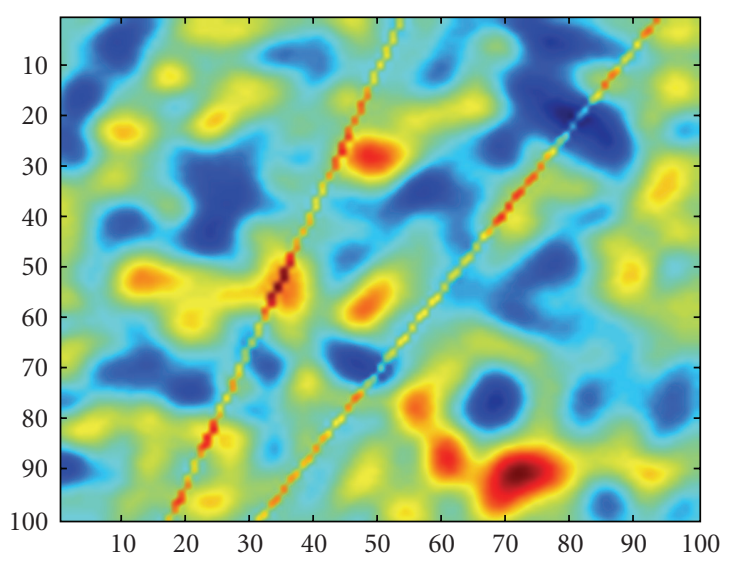

(a)

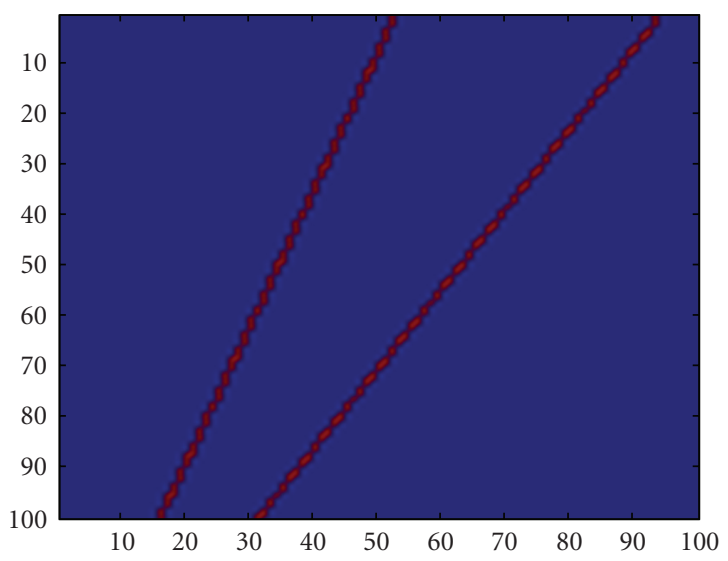

(c)

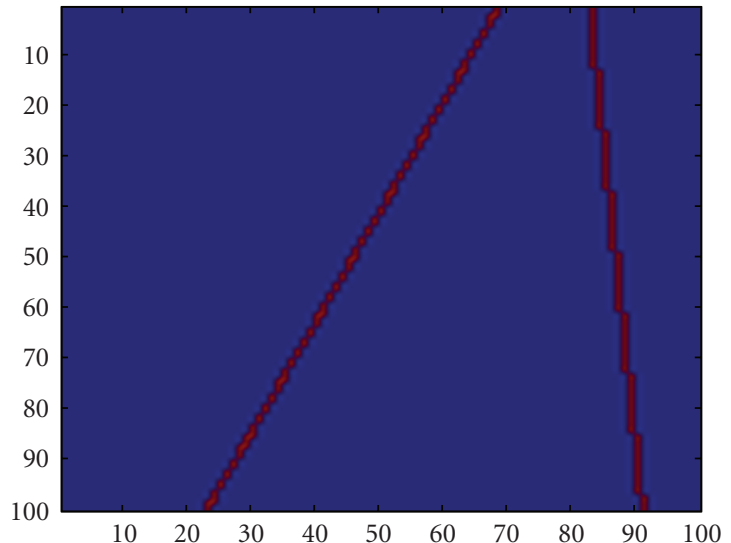

(b)

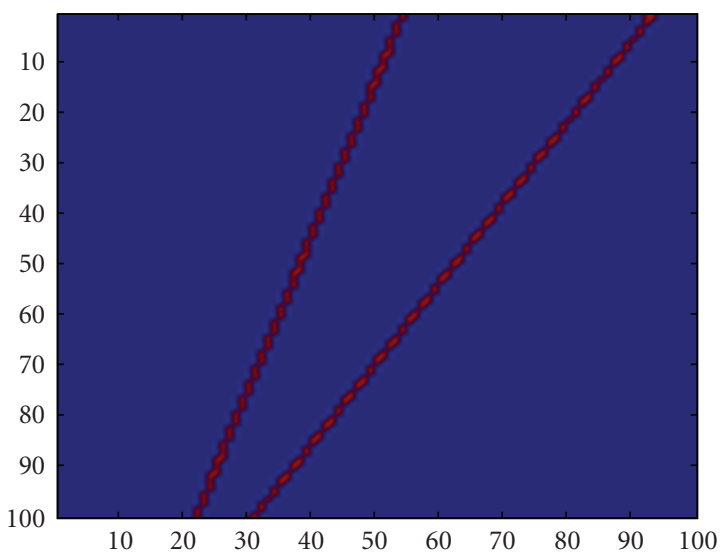

(d)

FIGURE 2: Initial image and estimated results for two lines with 32 and 20 degrees. (a) Initial scaled image. (b) Result without cumulant. (c) MUSIC-like algorithm. (d) TLS-ESPRIT algorithm.

$14.8^{\circ}$ and $45.3^{\circ}$, and they are $14.5^{\circ}$ and $45.7^{\circ}$ for improved TLS-ESPRIT algorithm. Then we change the angles to be $32^{\circ}$ and $28^{\circ}$, the mean values of estimated angles are $32.3^{\circ}$ and $27.9^{\circ}$ for 100 trials. For improved TLS-ESPRIT algorithm, we can also get the similar results, their means are $33.4^{\circ}$ and $29.1^{\circ}$.

Finally, to evaluate the computational load, we also compute the time elapsed to estimate the angle for the MUSIClike algorithm and improved TLS-ESPRIT algorithm. Our simulation run on the same system for two algorithms: Intel 2 Quad CPU, $2.83 \mathrm{GHz}$, with the memory $4 \mathrm{G}$. In the simulation, we set two lines with the angles $15^{\circ}$ and $45^{\circ}$ in the image. The time consumed by MUSIC-like algorithm is 0.29 seconds to obtain the estimations of angle values, while it is 0.10 seconds for improved TLS-ESPRIT algorithm.

\section{Conclusion}

In this paper, the problem of contour detection in image is investigated by using array processing. Considering the circumstance of correlated Gaussian noise, fourth-order cumulant-based MUSIC-like and improved TLS-ESPRIT algorithm for non-Gaussian virtual generated signal from the image are presented to estimate the orientations of lines, while the offsets of lines are estimated by "Extension of the Hough Transform." Numerical simulation shows that using cumulant-based algorithms improves the detection performance when correlated noise exists in image. These methods can also be extended to detect other contours in image, such as a curve line and a circle.

\section{References}

[1] R. O. Schmidt, "Multiple emitter location and signal parameter estimation," IEEE Transactions on Antennas and Propagation, vol. 34, no. 3, pp. 276-280, 1986.

[2] R. Roy and T. Kailath, "ESPRIT: estimation of signal parameters via rotational invariance techniques," IEEE Transactions on Acoustics, Speech, and Signal Processing, vol. 37, no. 7, pp. 984995, 1989.

[3] H. K. Aghajan and T. Kailath, "SLIDE: subspace-based line detection," IEEE Transactions on Pattern Analysis and Machine Intelligence, vol. 16, no. 11, pp. 1057-1073, 1994.

[4] S. Bourennane and J. Marot, "Coutour estimation by array processing methods," EURASIP Journal on Applied Signal Processing, vol. 2006, Article ID 95634, 15 pages, 2006.

[5] N. Yuen and B. Friedlander, "DOA estimation in multipath: an 
approach using fourth-order cumulants," IEEE Transactions on Signal Processing, vol. 45, no. 5, pp. 1253-1263, 1997.

[6] B. Porat and B. Friedlander, "Direction finding algorithms based on high-order statistics," IEEE Transactions on Signal Processing, vol. 39, no. 9, pp. 2016-2024, 1991.

[7] M. Wax and T. Kailath, "Detection of signals by information theoretic criteria," IEEE Transactions on Acoustics, Speech, and Signal Processing, vol. 33, no. 2, pp. 387-392, 1985.

[8] P. R. Thrift and S. M. Dunn, "Approximating point-set images by line segments using a variation of the Hough transform," Computer Vision, Graphics, and Image Processing, vol. 21, no. 3, pp. 383-394, 1983. 

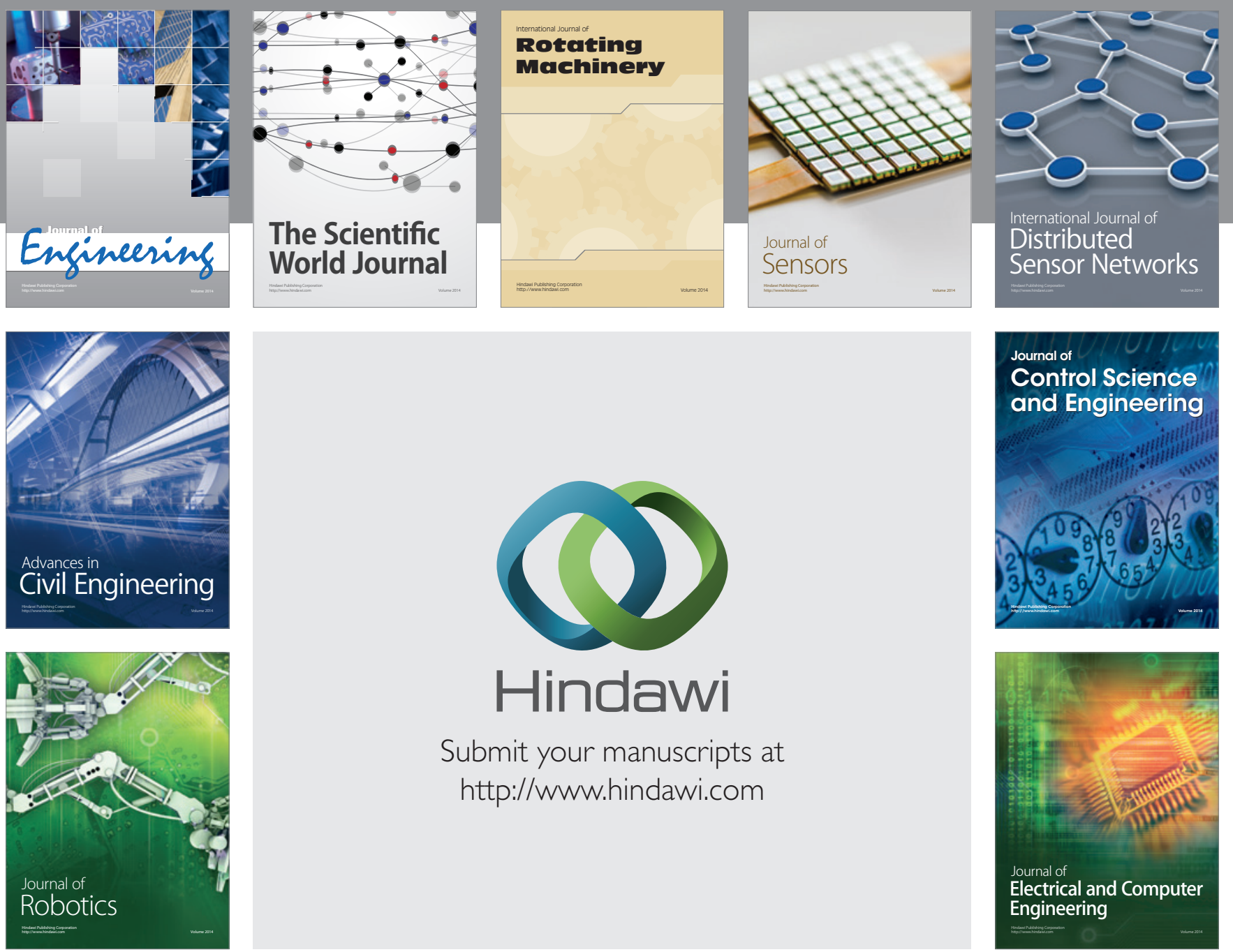

Submit your manuscripts at

http://www.hindawi.com
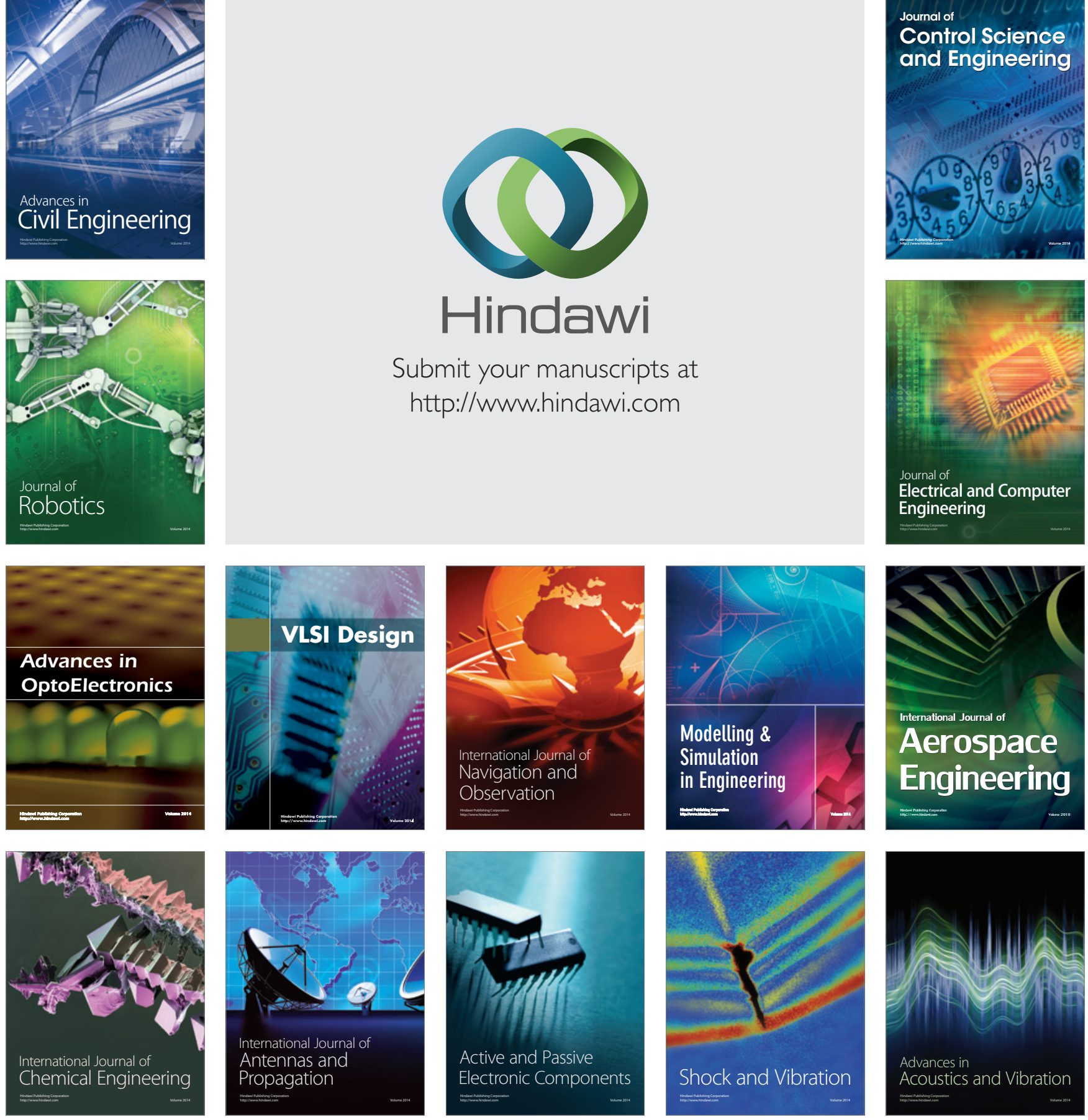